\title{
How Are Machine Learning Algorithms Different from Statistical Methods?
}

\section{Fu-Sheng Chou, MD, PhD}

Last month, we kicked off a new series of articles to introduce machine learning to the readers of Neonatology Today. The goal was to take a stepwise approach to deliver key terminology and concepts of this branch of artificial intelligence to the audience. This methodology is finding its way into neonatal and perinatal research. The hope is that when the readers come across manuscripts that involve this methodology for data analysis, there will already be a basic understanding of machine learning and what this term entails. In this issue, the objective is to provide a more in-depth discussion about the fundamental differences between statistical modeling and machine learning algorithms.

\section{"The hope is that when the readers come across manuscripts that involve this methodology for data analysis, there will already be a basic understanding of machine learning and what this term entails. In this issue, the objective is to provide a more in-depth discussion about the fundamental differences between statistical modeling and machine learning algorithms."}

\section{The bottom-up approach in statistical modeling}

In statistical modeling, a statistical approach is chosen based on the clinical question or, more often, based on the comfort level of the clinical researchers in the statistical approach selected for the analysis. Linear regression and logistic regression analyses are probably the most commonly utilized methods for the variable association. It usually starts with a "simple" regression analysis where one independent variable is attempted at a time to assess its correlation with the outcome (the dependent variable). As shown in Equation 1, the goal is to calculate "a." Based on our data, it is straightforward to calculate " $a$," but does our data represent the population? This is where statistics come in - to solve this uncertainty (of whether our data represents the universe) and provide an estimate for "a" to infer the population. A predefined $p$-value cutoff is used to determine whether " $a$ " can be zero or would always be a non-zero value, in which case the association between $x$ and $y$ can be considered as present or "statistically significant." Those variables $(x)$ that show significant associations individually with $y$ are then incorporated in a "multivariable" regression analysis, where selected variables (e.g., $x_{1}, x_{2}$, and $x_{3}$ in Equation 2) are added together on the right side of the equation. In contrast, the outcome variable remains on the left side. Statistics are then carried out again to assess whether $a_{1}, a_{2}$, and $a_{3}$ are likely non-zero. After the analysis, if $a_{2}$ is determined to be non-zero, we will then say, after adjusting for $x_{1}$ and $x_{3}$, that $x_{2}$ is independently associated with $y$.

$$
\begin{aligned}
& y=a x+b \\
& y=a_{1} x_{1}+a_{2} x_{2}+a_{3} x_{3}
\end{aligned}
$$

(Equation 2)

Most of our readers have experienced this analytic method numerous times. I am not trying to provide a statistics review here. Also, this is not the only way to build a statistical model; it is just an example. As one can see, humans select $x_{1}, x_{2}$, and $x_{3}$ for the multivariable analysis. One may have another equation used to estimate coefficients for $x_{1}$ and $x_{2}$ only and yet another equation that incorporates $x_{1}, x_{2}, x_{3}$, and $x_{4}$. Certain additional "goodness of fit" analyses are then performed to determine which equation fits the data and provides the best inference to the population. The final model is then born, and a manuscript is written up -- hoping to get published somewhere.

The statistical approach is a bottom-up approach, like building a house brick by brick, step by step, to assess the likely variables associated with the outcome. It is based on rational deduction according to clinical observations and hypotheses. We usually use it to study risk factors: for example, is high-grade intraventricular hemorrhage associated with worse neurodevelopmental outcomes after adjusting other risk factors?

\section{"The statistical approach is a bottom-up approach, like building a house brick by brick, step by step, to assess the likely variables associated with the outcome. It is based on rational deduction according to clinical observations and hypotheses."}

\section{The top-down approach in machine learning modeling}

The difference between statistics and machine learning is the "learning" part by the machines. Let us build upon the linear regression model to demonstrate how the machines "learn" from the data.

Here, two methods that have been applied on top of linear regression models allowing the machine to "learn" are 1) least absolute shrinkage and selection operator (LASSO) and 2) Ridge regression. It is commonly called "variable regularization." Without going into the formulas and mathematical details, and generally speaking, LASSO regression allows the machine to determine whether to "shrink" a coefficient for a variable down, even to 0 . When a coefficient approaches 0 , that variable is essentially negligible in the equation. In other words, when a coefficient is 0 , the machine has learned that this specific variable does not play a role in outcome prediction. The Ridge regression also shrinks the coefficients but, unlike LASSO, does not eliminate any variable. One can think of LASSO and Ridge as putting relative weights on each of the variables being tested. The goal is to minimize the difference between the predictive and actual outcomes and improve statistical inference.

There is an additional advantage for doing regularization. In lin- 
ear modeling, the researchers are most concerned about multicollinearity, where two or more independent variables are highly correlated. It leads to variable inflation and inaccurate estimation of the coefficients. LASSO and Ridge may take care of that. By adding additional terms, these methods put weights on each variable to reduce the impact of multicollinearity.

What does it mean by putting weights on each variable and reducing the impact of multicollinearity? It means that one does not have to hand-pick variables like the deductive approach in statistical model development. By learning from the data, the machine weighs the variables and determines which ones are important and which ones not as much. This is a top-down approach. We provide the predictors and the outcomes. The machines figure out the in-between.

\section{"By learning from the data, the machine} weighs the variables and determines which ones are important and which ones not as much. This is a top-down approach. We provide the predictors and the outcomes. The machines figure out the in-between."

\section{Hyperparameter}

How much LASSO and Ridge will attempt to shrink the variables is determined before data is fed into the algorithms. What controls how much to shrink is called a "hyperparameter." The coefficient for the penalty term that constrains the coefficients for the variables, called $\lambda$, is a hyperparameter. A hyperparameter always has to be pre-determined by humans before the machine starts to learn from the data. Feels good here, right? Humans control the hyperparameters, not the machines, so do not worry. At least in machine learning, machines are not taking over yet!

\section{The black-box fear}

In straightforward linear regression with manageable variable numbers, it is not too tricky to figure out the "in-between" by assessing how much weight each variable receives and rationalizing the why post hoc. In cases where there is an enormous number of observations and numerous variables for each observation, the "in-between" may start to overwhelm humans. This process is probably the fear clinicians have when talking about using machine learning-based models for augmenting clinical decision-making: how does the machine conclude that this baby will respond to indomethacin but not that one? Should one trust an educated guess, should one listen to the machine, or should one dig up that PDA treatment guideline that was developed ten years ago? And by the way, in this parallel world where individual prediction is available with sophisticated models, the flowchartbased protocol for PDA treatment is probably obsolete. After all, flowcharts cannot handle more than a handful of variables in their decision tree.

\section{Do NOT throw everything into the algorithm}

Before we conclude this topic, there is a key message that is very important for our readers. Machine learning is such a novel approach to neonatal research; researchers and clinicians may have the temptation to throw all variables they have collected over the years in a myriad of Excel files into the algorithms to see if these potential variables predict any outcome. Even though the researchers will not receive formal complaints from the machines because the machines cannot and will not complain, this is a dangerous approach and should not be encouraged in the literature. The reason is simple: humans develop machine learning because humans cannot handle excessive data. If humans put all the data they cannot handle together and feed this data into the machines, humans will soon be drowned, knowingly or unknowingly, by the data the engines put out on the other end of the algorithm. Machines "augment" the decision-making process owned by humans; they do not take over the process. Indiscriminate data entry leads to indeterminate results. Please do not assume that one can input all the available data, and the machine will deliver a guidebook or a policy statement to solve clinical questions. Machine learning-based predictive model development should follow the well-established steps developed by experts in the field, just like statistical models should be developed. The steps may be different, but the core spirit is the same.

\section{Ask the machine to develop clinically relevant models}

It is also important to be mindful about what outcomes to predict. Are they clinically relevant? During this COVID-19 pandemic, many chest $\mathrm{X}$-rays and chest CT scans were performed on patients to determine the severity and monitor radiographic response. With access to these image data, researchers hoped to use a branch of machine learning called deep learning to investigate whether image studies can be used to replace or augment COVID-19 diagnosis. In other words, can a machine learning model be developed to distinguish between pneumonia caused by COVID-19 or by other etiologies? This seemingly plausible idea goes against the definition of pneumonia in the first place and makes no sense in clinical management. As we learned in medical school, pneumonia is a clinical diagnosis, not a radiographic diagnosis. It is diagnosed by history and physical exam. We should never start a machine learning project just because we have the data.

\section{"Machine learning-based predictive model development should follow the well- established steps developed by experts in the field, just like statistical models should be developed. The steps may be different, but the core spirit is the same."}

\section{Conclusion}

Unlike rigorous hypothesis-driven research, machine learningbased research is more about outcome prediction, exploration of novel variables, and comparing the performance of different algorithms. The predictors are usually already known to have a potential association with the outcome, albeit can be assumed loosely and anecdotally, or are tested for hypothesis generation. These two goals are sometimes combined with learning about novel variables and then using these novel variables to predict the outcomes. Going back to the point that was made earlier, do not throw every variable into the algorithm and wish that magic will show. It will not, unfortunately.

I hope readers new to machine learning feel a little bit relieved by this brief comparison between statistics and machine learning. Machine learning is still mathematics and statistics. It incorporates 
additional steps to optimize outcome association systemically. These extra steps are what is perceived as "learning." There are different algorithms in machine learning, many of which are based on non-linear approaches. We will talk more about these other algorithms next month.

\section{Disclosure: The author identifies no conflict of interest}

NT
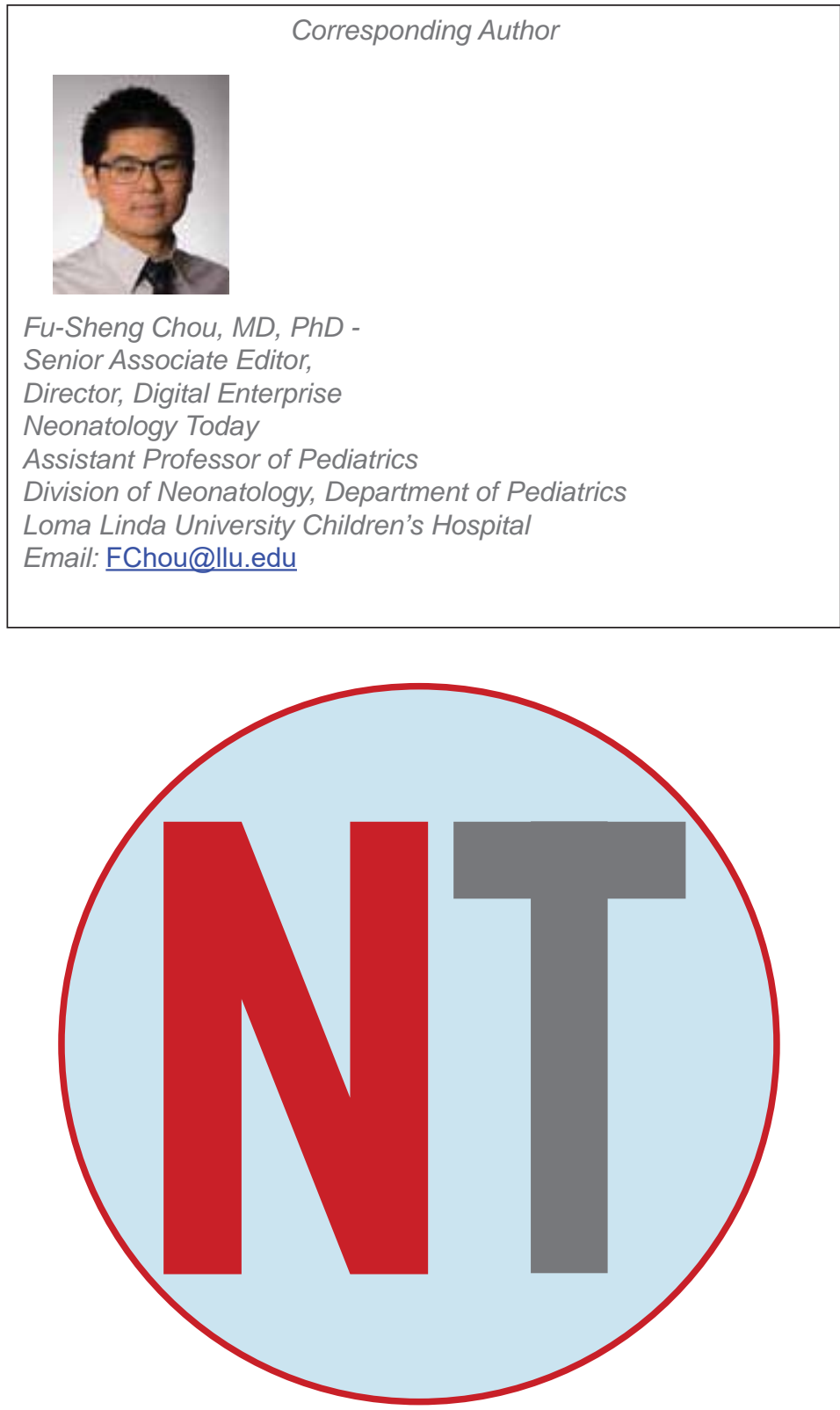

\section{Neonatology Today's Digital Presence}

Neonatology Today's now has a digital presence. The site is operational now and defines the future look of our digital web presence. By clicking on this https://www.neonatologytoday.org/ web/., researchers can download individual manuscripts both in digital format and as part of the original PDF (print journal). While the PDF version of Neonatology Today will continue in its present form, we envision that the entire website will be migrated to this format in the next several months. We encourage you to take a look, "kick the wheels," and let us know where we still need to improve.. We are working towards making the website more functional for subscribers, reviewers, authors and anyone else. Although we have not yet applied for inclusion in the National Library of Medicine Database (Pub-Med), this new format meets several of the important metrics for this ultimate goal. As of December, 2020, NT has its own account with CrossRef and will assign DOI to all published material.

As we indicated last month, we look forward to a number of new features as well.

1. An online submission portal: Submitting a manuscript online will be easier than before. Rather than submitting by email, we will have a devoted online submission portal that will have the ability to handle any size manuscript and any number of graphics and other support files. We will have an online tracking system that will make it easier to track manuscripts in terms of where they are in the review process.

2. Reviewers will be able to review the manuscript online. This portal will shorten the time from receipt of review to getting feedback to the submitting authors.

3. An archive search will be available for journals older than 2012.

4. A new section called news and views will enable the submission of commentary on publications from other journals or news sources. We anticipate that this will be available as soon as the site completes the beta phase

5. Sponsors will be able to sign up directly on the website and submit content for both the digital and PDF issues of Neonatology Today.

Neonatology Today will continue to promote our Academic True Open Model (ATOM), never a charge to publish and never a charge to subscribe.

If there are any questions about the new website, please email Dr. Chou directly at:

fu-sheng.chou@neonatologytoday.net

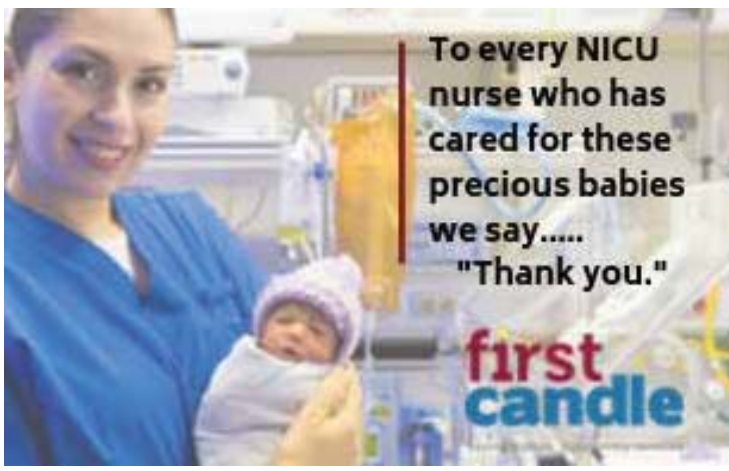

\section{Did you know that premature and low birth weight babies have a $4 x$ greater risk for SIDS?}

At First Candle we're educating parents, grandparents and caregivers about safer sleep to make sure all babies reach their first birthday. Learn more at firstcandle.org 